\title{
Novel tumor suppressive function of Smad4 in serum starvation-induced cell death through PAK1-PUMA pathway
}

\author{
S-H Lee ${ }^{1}$, Y-S Jung ${ }^{1}$, J-Y Chung ${ }^{1}$, AY Oh ${ }^{1}$, S-J Lee ${ }^{1}$, DH Choi ${ }^{2}$, SM Jang ${ }^{3}$, K-S Jang ${ }^{3}$, SS Paik ${ }^{3}$, N-C Ha ${ }^{4}$ and B-J Park ${ }^{* 1}$
}

DPC4 (deleted in pancreatic cancer 4)/Smad4 is an essential factor in transforming growth factor (TGF)- $\beta$ signaling and is also known as a frequently mutated tumor suppressor gene in human pancreatic and colon cancer. However, considering the fact that TGF- $\beta$ can contribute to cancer progression through transcriptional target genes, such as Snail, MMPs, and epithelialmesenchymal transition (EMT)-related genes, loss of Smad4 in human cancer would be required for obtaining the TGF- $\beta$ signaling-independent advantage, which should be essential for cancer cell survival. Here, we provide the evidences about novel role of Smad4, serum-deprivation-induced apoptosis. Elimination of serum can obviously increase the Smad4 expression and induces the cell death by p53-independent PUMA induction. Instead, Smad4-deficient cells show the resistance to serum starvation. Induced Smad4 suppresses the PAK1, which promotes the PUMA destabilization. We also found that Siah-1 and pVHL are involved in PAK1 destabilization and PUMA stabilization. In fact, Smad4-expressed cancer tissues not only show the elevated expression of PAK1, but also support our hypothesis that Smad4 induces PUMA-mediated cell death through PAK1 suppression. Our results strongly suggest that loss of Smad4 renders the resistance to serum-deprivation-induced cell death, which is the TGF- $\beta$-independent tumor suppressive role of Smad4.

Cell Death and Disease (2011) 2, e235; doi:10.1038/cddis.2011.116; published online 1 December 2011

Subject Category: Cancer

Human cancer progression is driven by accumulation of genetic mutation. ${ }^{1}$ According to the multistep carcinogenesis model of pancreatic and colon cancer, ${ }^{2,3}$ oncogenic mutation of K-Ras, p16 inactivation by methylation or deletion, and Smad4 deletion occur in pre-carcinoma stage. ${ }^{4,5}$ Considering the fact that Smad4 deletion or mutation occurs in juvenile polyposis coli, ${ }^{6}$ it is clear that Smad4 exerts the critical tumor suppressor function. In previous, we have suggested that oncogenic K-Ras, which is mutated in early adenoma stage, can suppress the p53 function. ${ }^{7,8}$ Thus, cell proliferation ability of oncogenic K-Ras may be appeared in pre-cancerous pancreatic epithelial cells. In addition, p16 inactivation can render the resistance to transforming growth factor (TGF)- $\beta$-induced or pRB-mediated cell cycle inhibition. ${ }^{9,10}$ Hence, further genetic mutations of TGF- $\beta$ signaling components seem to be not essential for cancer progression. Moreover, Smad4, but not T $\beta \mathrm{RI}$, II, or $\mathrm{R}-\mathrm{Smad}$ is frequently mutated in human colon, and pancreatic cancer has not been clearly demonstrated. As TGF- $\beta$ can also contribute to epithelial-mesenchymal transition (EMT) progression through transcriptional target genes, such as Snail, $M M P s$, and EMT-related genes, ${ }^{11,12}$ it seems to be not reasonable that cancer cells inactivate Smad4 to gain the resistance to TGF- $\beta$ signaling. Instead, loss of Smad4 in human cancer would provide that TGF- $\beta$ signaling-independent advantage, which should be essential for cancer cell survival.

During colon or pancreatic cancer progression, Smad4 deletion occurs at late hyperplasia or carcinoma stage, where tumor volume is increased. ${ }^{2,3}$ Thus, inner cell mass of tumor would be suffered by nutrient deprivation and hypoxia. To overcome this, cancer cell should develop the defense mechanism. One of plausible and required event seems to be inactivation of apoptosis mechanism. In this study, we investigate the relevance of Smad4 and nutrient-deprivation-induced apoptosis. Here, we provide the several evidences about involvement of Smad4 in serum starvation-induced cell death, which is mainly achieved by induction of PUMA. Although PUMA is an immediate-early target gene of $p 53,{ }^{13,14}$ we find that induction of PUMA is achieved by $p 53$-independent mechanism. Instead, Smad4 induces PUMA through suppression of PAK1, which is elevated in Smad4-positive human colon cancer tissues. Our results suggest that Smad4 exerts the novel tumor suppressive function through PUMA induction and PAK1 is worked as intrinsic inhibitor against Smad4-induced cell death.

\footnotetext{
${ }^{1}$ Department of Molecular Biology, College of Natural Science, Pusan National University, Busan, Republic of Korea; ${ }^{2}$ Department of Surgery, Soonchunhyang Medical Center, Soonchunhyang University, Seoul, Republic of Korea; ${ }^{3}$ Department of Pathology, College of Medicine, Hanyang University 17, Haengdang-dong, Seongdong-gu, Seoul 133-792, South Korea and ${ }^{4}$ Department of Manufacturing Pharmacy, College of Pharmacy, Pusan National University, Busan, Republic of Korea ${ }^{*}$ Corresponding author: B-J Park, Department of Molecular Biology, College of Natural Science, Pusan National University, Busan, Republic of Korea. Tel: + 8251510 2220; Fax: + 8251513 9258; E-mail: bjpark1219@ pusan.ac.kr Keywords: DPC4/Smad4; colon cancer; PUMA; PAK1

Abbreviations: TGF- $\beta$, transforming growth factor- $\beta$; EMT, epithelial-mesenchymal transition; SF, serum free; IP, immunoprecipitation; DN-E-cad, dominant-negative E-cadherin

Received 28.6.11; revised 12.9.11; accepted 21.9.11; Edited by A Stephanou
} 
a

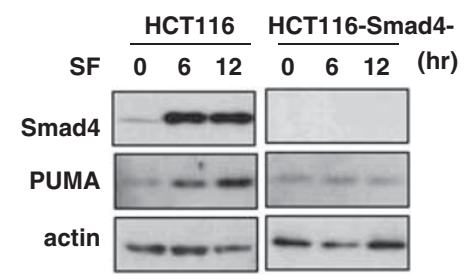

C

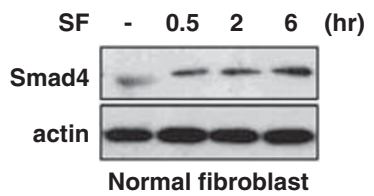

e

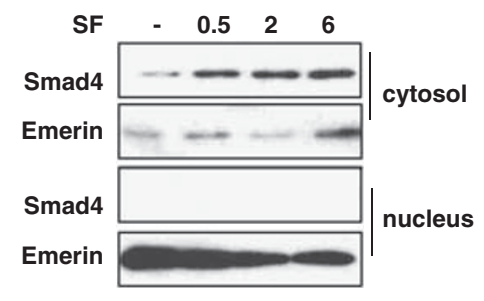

f

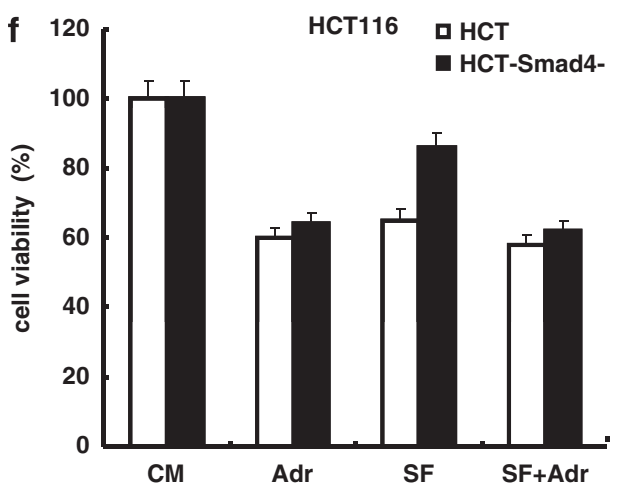

b

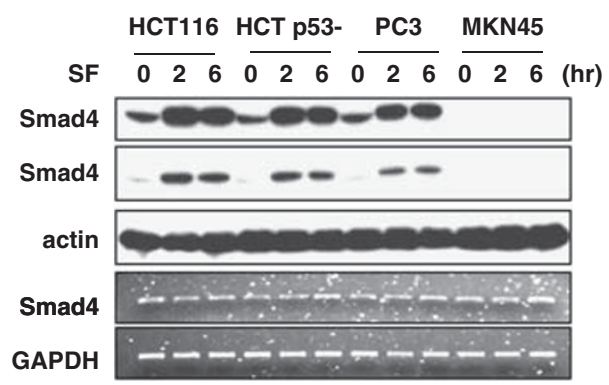

d

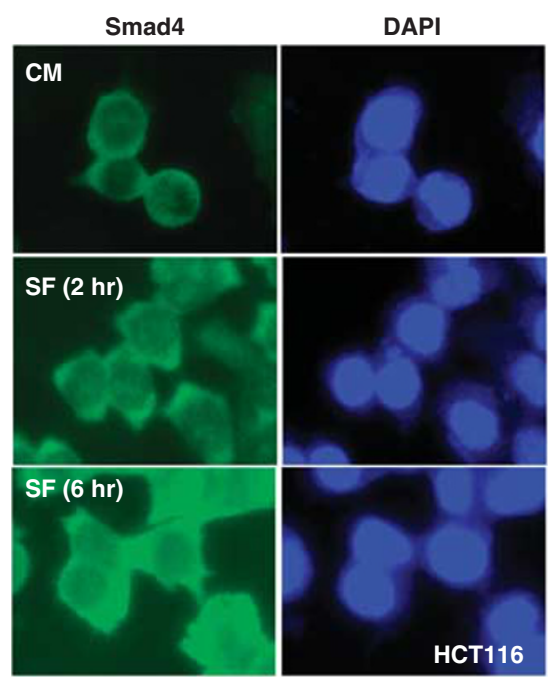

Figure 1 Induction of Smad4 in response to serum starvation. (a) Induction of Smad4 and PUMA in response to SF condition. HCT116 and its isogenic Smad4-deficient cells were incubated in SF condition for indicating time. Actin was used as loading control. (b) Serum starvation leads to Smad4 induction in three kinds of cell lines, but not in MKN45, at post-transcription level. Smad4 expression was determined by WB analysis at translation level and by reverse transcription (RT)-PCR analysis at transcription level. (c) Induction of Smad4 is observed in normal fibroblast. Normal human fibroblast was exposed to SF condition for indicating time, and the Smad4 expression level was evaluated by WB. (d) Serum starvation-induced Smad4 is accumulated in cytosol. After fixation with Me-OH, cells were stained with anti-Smad4 (green) and 4'6-diamidino-2phenylindole (DAPI; blue). (e) Smad4 is retained in cytosol. HCT116 cells were divided into four subcellular fractions using a Sub cell kit (Calbiochem), and the expressions of Smad4 were examined. (f) Smad4-deficient HCT116 cells showed resistance to SF condition compared with positive cells, but in the presence of DNA-damaging agent, adriamycin (Adr; $2 \mu \mathrm{g} / \mathrm{ml})$, both cells revealed similar sensitivity. Cell viability was determined by MTT assay

\section{Results}

Induction of Smad4 in response to serum starvation. To address the role of Smad4 during cancer progression, we investigated the expression of Smad4 in colon cancers and found that Smad4 reduction occurs at transition stage from adenoma to carcinoma (Supplementary Figure 1a, Supplementary Table 1). As carcinoma cells are resistant to nutrient and oxygen deprivation, we tested the role of Smad4 in response to serum starvation and hypoxic stress. Interestingly, Smad4 expression was obviously induced by serum starvation, but not by hypoxic stress (Supplementary Figure 1b). We also measured the expression of p53, p21 and PUMA, because serum starvation and hypoxic stress can activate p53. Although p53 and p21 were induced by hypoxic stress, PUMA showed the Smad4-dependent manner (Supplementary Figure 1b). We also measured the expression of Smad4 and PUMA under serum-free (SF) condition in HCT116 and its isogenic Smad4-deficient cells, and obtained the same result that PUMA induction was achieved by Smad4-dependent manner (Figure 1a). We also checked the Smad4 expression in four kinds of human cancer cell lines, and found that Smad4 in three kinds of cell lines were induced by serum starvation at posttranscription level (Figure 1b). From the extended study, we found that Smad4 induction was a general event for cell lines, except for some kinds of cell lines, such as MKN45 and SK-N-SH (Supplementary Figure 1c). As MKN45 is a gastric cancer cell line, we checked the Smad4 induction in gastric cell lines. However, all of tested cell lines, except MKN45, showed the response to serum starvation (Supplementary Figure 1d). Next, we monitored the Smad4 expression in neuron cell and leukemia cell line. In these 
a
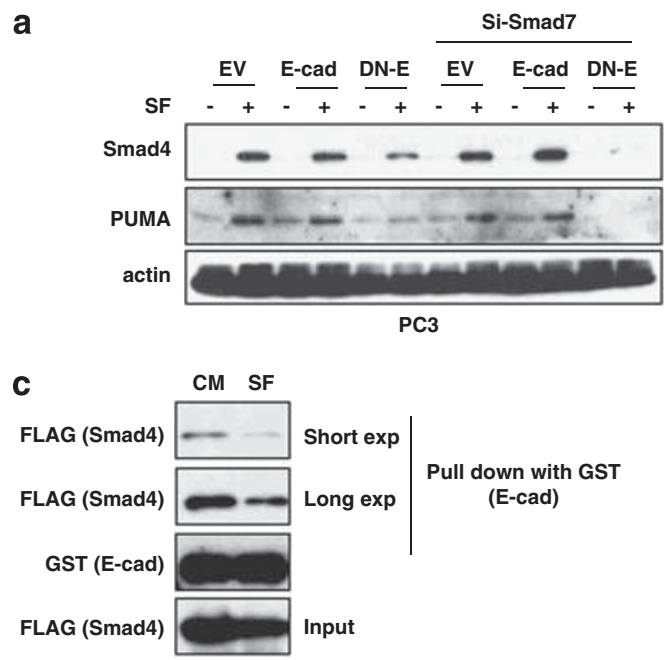

b

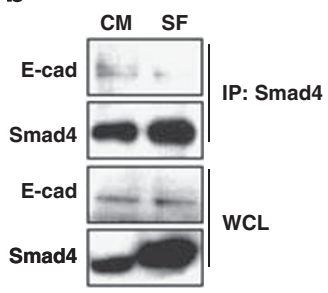

d

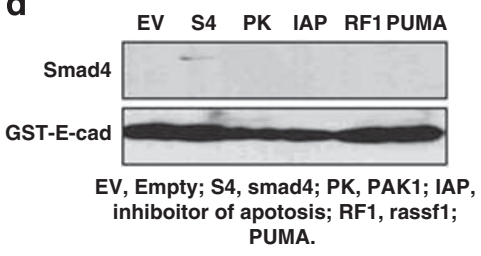

e

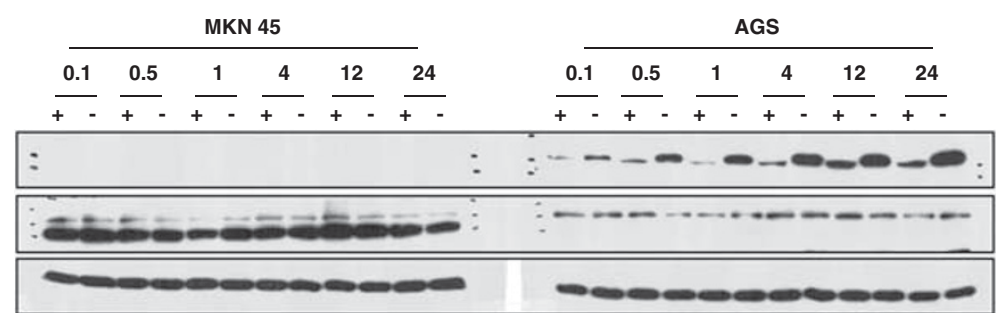

Figure 2 E-cad is involved in Smad4 regulation. (a) Blocking the E-cad reduces the Smad4 induction in response to SF condition. PC3 cells were transfected with empty vector (EV), E-cad, DN-E-cad (DN-E), and siRNA Smad7. (b) Interaction between Smad4 and E-cad was decreased in SF condition. At $24 \mathrm{~h}$ after transfection, HEK293 cell lysates were immunoprecipitated with anti-Smad4 and analyzed by WB. (c) Smad4 is released from E-cad in SF condition. GST-E-cad was incubated with Smad4-FLAGtransfected HEK293 cell lysates in RIPA buffer for $2 \mathrm{~h}$. After washing, precipitated protein was subjected to SDS-PAGE and WB with the indicated antibodies. (d) Interaction between Smad4 and E-cad is a specific event. GST pull down-was performed with several proteins, and each protein was analyzed by detectable antibodies (data not shown). (e) In the serum-deficient condition, Smad4 induction is only shown in AGS cells (containing wild-type E-cad), but not in mutant E-cad harboring MKN45. Each cell line was exposed to SF condition for indicated times, and Smad4 and E-cad proteins were detected by WB

cell lines, we did not observe the induction of Smad4 including K562 (Supplementary Figures 1e and f). We could observe the induction of Smad4 in response to serum starvation in normal cell (Figure 1c), indicating that SF condition-induced Smad4 is a general event. As Smad4 is a well known transcription factor, we determined the localization of Smad4 through immunofluorescence staining and cell fractionation assay. However, serum-deprivationinduced Smad4 was retained in cytosol (Figures 1d and e). To address the biological meaning of Smad4 induction, we measured the cell viability of HCT116 and HCT116 Smad4-/-cell lines ${ }^{15}$ in SF condition and found that Smad4-/- cell showed the resistance to SF conditioninduced cell death, whereas these cell lines showed the similar response to DNA damaging agent, adriamycin (Figure 1f). This result indicates that Smad4 would be not related with DNA damage-induced cell death pathway.

TGF- $\beta$ signaling-independent Smad4 induction by serum starvation. To reveal the induction mechanism of Smad4, we checked the involvement of TGF- $\beta$ signaling. Blocking of $\mathrm{R}$-Smad using siRNA or activation TGF- $\beta$ signaling, using constitute active $\mathrm{T} \beta \mathrm{R} 1$ transfection, did not alter the Smad4 expression (Supplementary Figures $2 \mathrm{a}$ and b). In addition, overexpression of inhibitory Smad, Smad7, or treatment of TGF- $\beta$ neutralizing antibody could not block the SF-induced
Smad4 expression (Supplementary Figures $2 \mathrm{~b}$ and c). Other kinds of TGF- $\beta$ signaling components did not also show the relevance with Smad4 induction by SF condition (Supplementary Figure 2d). As human or bovine serum contains growth factors, we checked the other possibility such as growth factor deprivation. However, IGF-1 did not block the Smad4 induction by serum starvation (Supplementary Figure 3a). In addition, mitochondria ATP synthesis blockers (cyclosporin A and oligomycin) did not induce Smad4 in serum-present condition, indicating that induction of Smad4 would not be triggered by energy deprivation (Supplementary Figure 3b). Instead, purified BSA could block the induction of Smad4 (Supplementary Figure 3c). These results indicate that extracellular nutrient concentration is critical for Smad4 induction.

E-cadherin regulates SF-induced Smad4. As E-cadherin (E-cad)-mutated MKN45 ${ }^{16}$ and leukocytes, which do not express E-cad, ${ }^{17}$ did not respond to SF (Figure $1 \mathrm{~b}$, Supplementary Figures 1e and f), we speculated that E-cad is involved in Smad4 induction. Consistent with our hypothesis, dominant-negative (DN)-E-cad could block the SF induction of Smad4 as well as PUMA (Figure 2a). Hence, we tested the interaction between Smad4 and E-cad, and revealed that Smad4 was released from E-cad under SF condition by immunoprecipitation (IP) and GST pull-down 
a

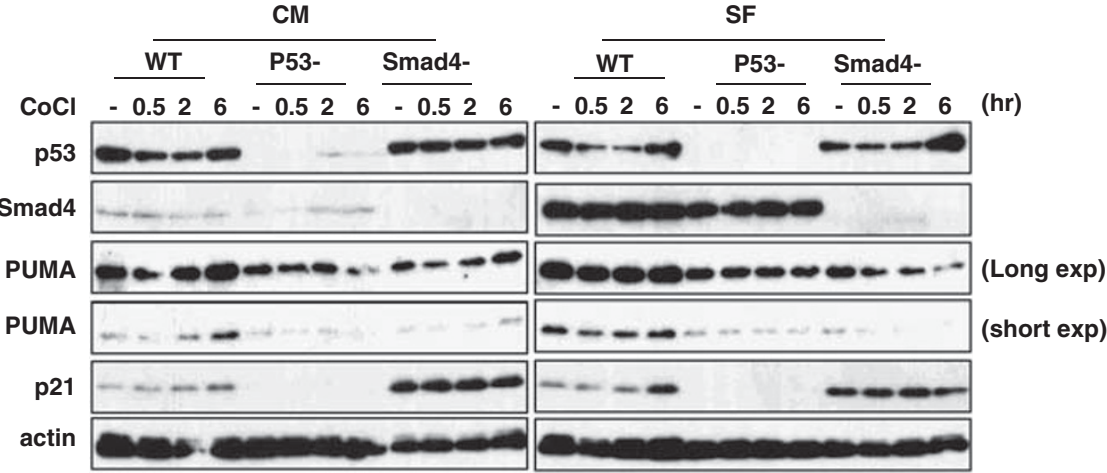

b

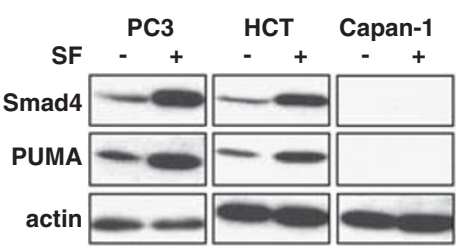

e

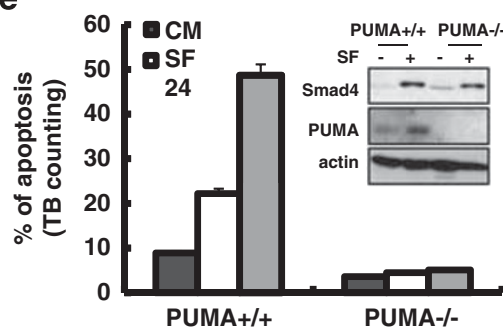

f

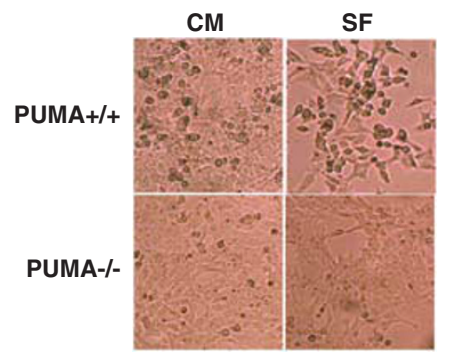

C

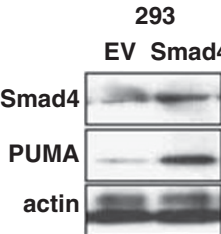

g

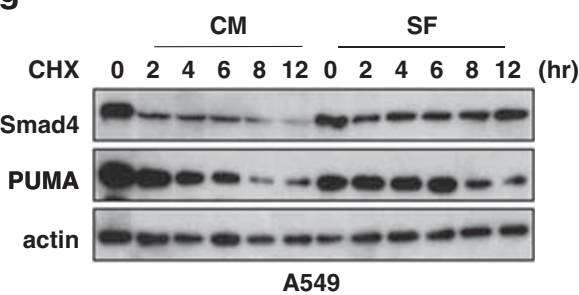

h

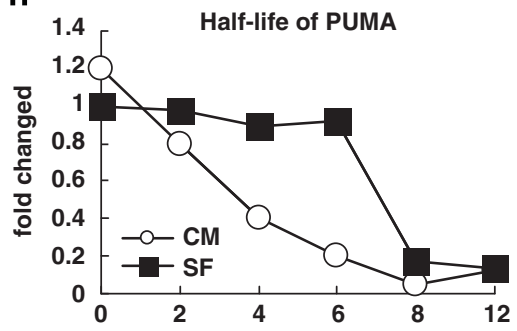

Figure 3 Smad4 induces PUMA. (a) PUMA is induced by Smad4 in response to SF condition. PUMA expression level is significantly decreased not only in p53-deficient cells but also in Smad4-deficient cells. Expression of indicated proteins in HCT116 cells was assessed by WB. (b) Smad4-dependent induction of PUMA. PUMA induction was not detected in Smad4-deficient Capan-1 cells. (c) Overexpression of Smad4 induces PUMA expression. HEK293 cells were transfected with empty vector (EV) and Smad4, and indicating proteins were detected by WB analysis. (d) Smad4 transfection can restore the SF-induced cell death in Smad4-deficient Capan-1. Cells were transfected with EV and Smad4 for $24 \mathrm{~h}$, and viability in different serum concentration was determined by MTT assay. (e and f) Absence of apoptosis in PUMA-deficient HCT116. HCT116 (PUMA + I+ ) and its isogenic PUMAdeficient cells (PUMA-I-) were incubated in SF condition for 24 or $48 \mathrm{~h}$ (f). Dying cells were counted using trypan blue dye exclusion assay. PUMA deficiency did not alter the Smad4 induction by SF condition (inset). (g) Extension of protein half-life of Smad4 and PUMA in SF condition. A549 cells were incubated in SF condition for $6 \mathrm{~h}$ to adjust the starting protein levels and were incubated in SF or complete media condition for indicated time with CHX $(100 \mu \mathrm{g} / \mathrm{ml})$. (h) The half-life of the PUMA protein is remarkably increased in SF condition. Expression level of PUMA was determined by densitometer. Presented graph showed the fold changes compared with control (CHX 0 time in SF condition)

assay (Figures $2 \mathrm{~b}$ and $\mathrm{c}$ ). To confirm this, we performed the GST pull-down again with several kinds of proteins and found that Smad4-E-cad binding was specific (Figure 2d). In fact, serum starvation-induced Smad4 was not detected in MKN45 but in AGS cell (wild-type E-cad; Figure 2e). These results indicate that SF-induced Smad4 is achieved by dissociation from E-cad and provide the explanation about the insensitivity of Smad4 induction in neural cells, which possesses $\mathrm{N}$-cadherin and leukemia cell lines (Supplementary Figures 1e and f).

Smad4 induces PUMA. Next, we focused on induction of PUMA as the cell death mechanism (Figure 1a). As PUMA 
a

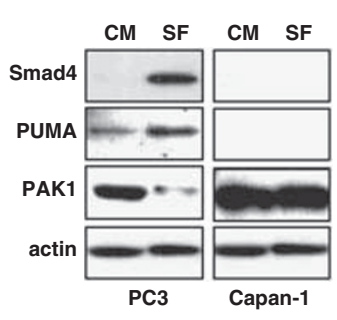

b

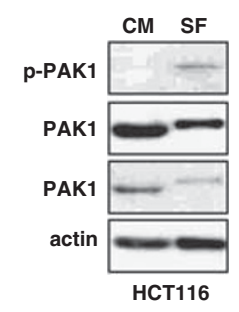

C
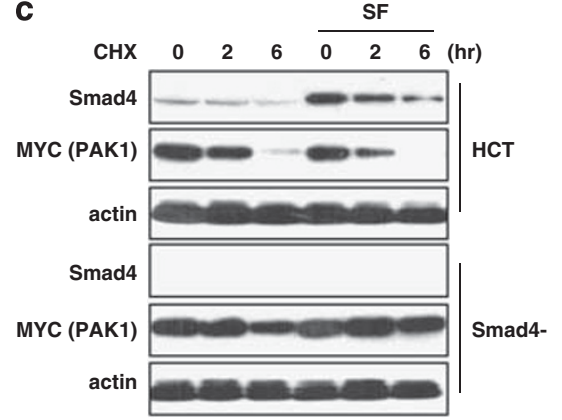

e PAK1

Smad4

PAK1

Si-Cont Si-Smad4

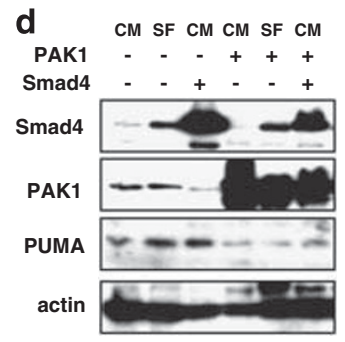

PUMA

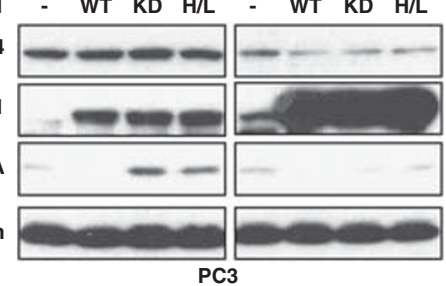

f

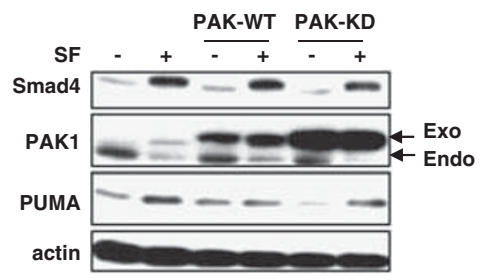

Figure 4 Smad4 regulates PUMA through PAK1. (a) PAK1 is reduced in SF condition. Cells were incubated with SF condition for $6 \mathrm{~h}$. (b) In response to serum starvation, phoshorylated PAK1 (p-PAK1) is increased, whereas total PAK1 is reduced. HCT116 cells were incubated with SF condition for $6 \mathrm{~h}$. (c) PAK1 expression is suppressed by Smad4. Compared with Smad4-deficient HCT116 cells, Smad4 stability was increased, whereas PAK1 expression was reduced in response to SF. CHX was treated with each cell line, and Smad4 and PAK1 (Myc tagged) expression levels were examined by WB analysis. (d) Smad4 overexpression induces PUMA via suppression of PAK1 expression. However, elevated PAK1 expression could attenuate Smad4-mediated PUMA induction in SF condition. HEK293 was transfected with wild-type PAK1 and Smad4 for $24 \mathrm{~h}$. (e) Knockdown of Smad4 using siRNA-induced PAK1 expression. siRNA Smad4, wild-type PAK1, kinase-dead PAK1 (KD-PAK1; K299R), and H/L (H83LH86L; GTPase binding domain mutant) was transfected to PC3 for $24 \mathrm{~h}$, and indicated proteins were measured by WB. (f) PAK1 mutants could not suppress Smad4-mediated PUMA induction in SF condition. Expression of PUMA was repressed by wild-type PAK1 but not by KD-PAK1

has been identified as $p 53$ target gene, ${ }^{13,14}$ we checked the expression of PUMA in p53- and Smad4- HCT116 cell lines. Consistent with previous reports, PUMA expression was low in p53-deficient HCT116 (Figure 3a). However, PUMA expression was also reduced in Smad4-deficient cell line (Figure $3 \mathrm{a}$ ). In addition, it could be induced by serum starvation for $6 \mathrm{~h}$ in p53-deficient cell but not in Smad4 cell (Figure 3a). To confirm it, we measured the expression of Smad4 and PUMA in PC3 (p53-), HCT116 and Capan-1 (Smad4-) under SF condition and obtained the similar result that induction of PUMA by SF condition is not related with p53 but Smad4 (Figure 3b). We also observed the PUMA induction by Smad4 transfection alone (Figure 3c). To extend this, we measured the expression of PUMA in several kinds of cell lines, including Smad4-deleted MDA-MB 468 and p53-null PC3. In this experiment, we could observe the induction of PUMA only in PC3, HCT116, and 293 cell lines, but not in MKN45 and MDA-MB 468 (Smad4-), by SF treatment. In addition, despite of induction of Smad4, MIAPaca-2 and Panc-1 did not show the induction of PUMA (Supplementary Figure 4a). These results indicate that although PUMA induction was achieved by Smad4, there would be additional regulation or inhibition mechanism between Smad4 and PUMA. To exclude the relevance of p53 in SF-induced PUMA, we monitored the expression of PUMA after treatment of DNA-damaging agents (adriamycin and etoposide) in HCT116 and HCT116 p53-/- cells. Comparing with induction of PUMA by DNA-damaging agents, more obvious induction of PUMA was detected in p53-/- cells under SF condition (Supplementary Figure 4b). We also measured the PUMA expression in various kinds of p53 mutant-transfected cells. However, PUMA induction was apparently increased by SF condition, regardless of p53 mutants (Supplementary Figure 4c). To verify the role of Smad4 in SF-induced cell death, we transfected the Smad4 into Capan-1 (Smad4- cell) and monitored the cell viability. Comparing with EV-transfected cells (the marginal suppression of cell viability), viability of Smad4-transfected cells was obviously reduced (Figure $3 d$ ). To get more clear evidence, we measured the cell viability in PUMA-deficient HCT $116^{18}$ using trypan blue dye exclusion assay and found that PUMA is essential for cell death in SF condition (Figures $3 e$ and $f$ ). To examine the regulation mechanism, we measured the transcript of PUMA. However, serum starvation did not induce the PUMA transcript or promoter activity (Supplementary Figures 5a and b). Instead, 

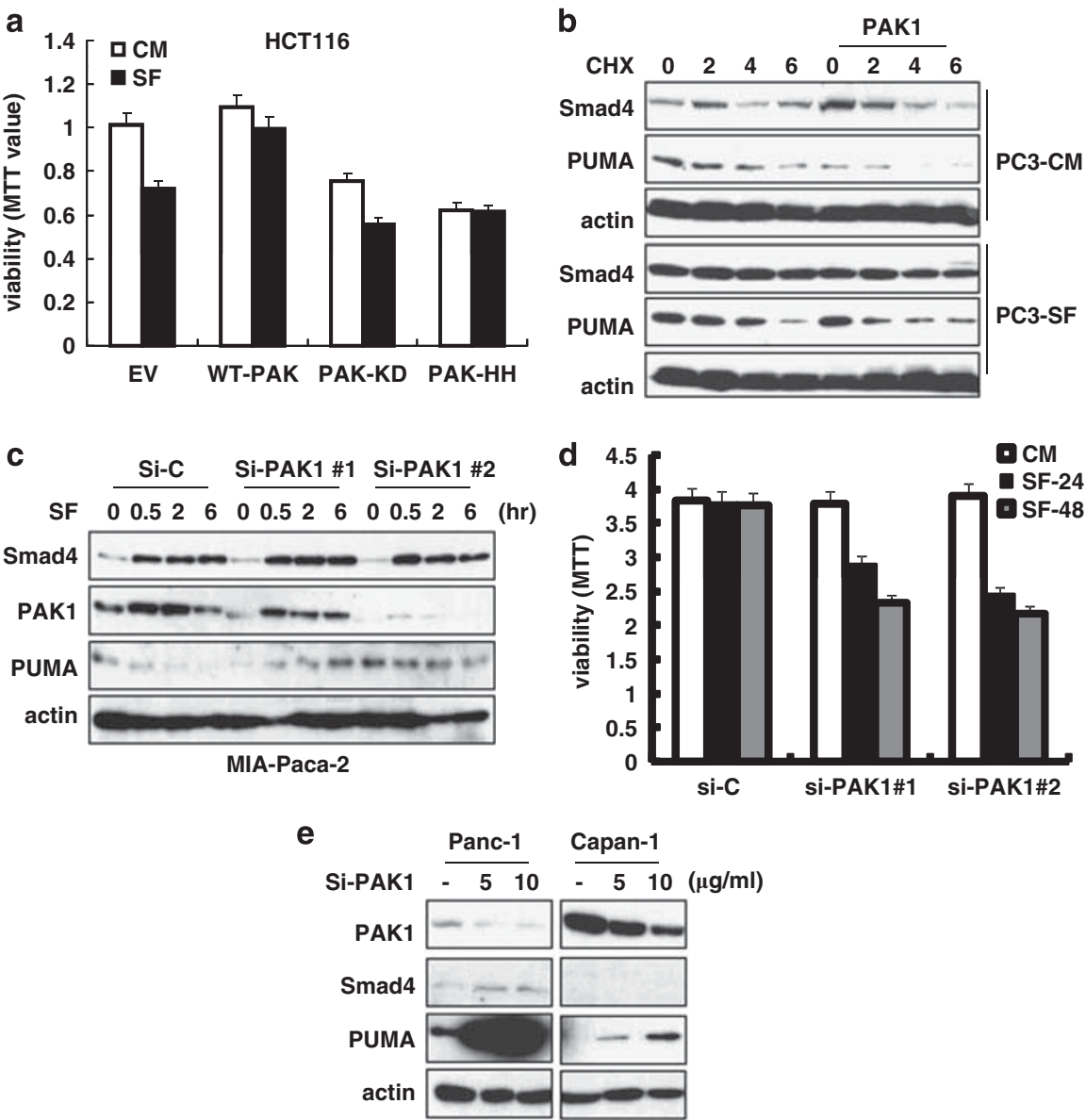

Figure 5 PAK1 regulates SF-induced cell death and PUMA expression. (a) Serum deprivation-induced cell death is reduced by wild-type PAK1, but not by mutant PAK1. Cell viability in SF condition was checked by MTT assay in HCT116 after transfection with PAK1s. (b) The stability (half-life) of PUMA is decreased by overexpression of wildtype PAK1. PC3 cells were transfected with wild-type PAK1, and cell lysates were analyzed by WB after incubating with CHX. (c) PAK1 knockdown restores PUMA induction in response to SF condition. MIA-Paca-2 cells were transfected with si-PAK1 for $24 \mathrm{~h}$, and expressions of indicated proteins were evaluated by WB analysis. (d) Elimination of PAK1 increases the sensitivity to serum starvation-induced cell death. siRNA PAK1 no. 1 and no. 2 were transfected for $24 \mathrm{~h}$ before exposing to SF condition. Cell viability was determined by MTT assay. (e) PAK1 knockdown induces PUMA expression in pancreatic cancer cell lines. Cells were transfected with a range of si-PAK1 concentration for $24 \mathrm{~h}$, and PUMA induction by disruption of PAK1 is revealed even in Smad4-null cell, Capan-1. Expression of indicating proteins were assessed by WB

PUMA expression was increased in cytosol (Supplementary Figure 5c). Hence, we next checked the PUMA protein stability. Through pulse-chase analysis, we found that protein stability of PUMA and Smad4 was obviously extended by serum starvation (Figures $3 g$ and $h$ ). This result may provide the evidence that Smad4 regulation was achieved at posttranscription level. We could confirm it using PC3 and HCT116 cell lines (Supplementary Figures $5 d-f$ ). In fact, Smad4 half-life was extended from $2 \mathrm{~h}$ to up to $10 \mathrm{~h}$, and PUMA half-life was also extended about twofolds (Figure $3 \mathrm{~g}$ and Supplementary Figure 5f). To address the molecular mechanism about Smad4-induced PUMA stabilization, we tested the interaction between PUMA and Smad4. However, we did not observe the interaction between them (Supplementary Figure $5 \mathrm{~g}$ ). Hence, we checked the effect of ER stress because it can induce PUMA expression in a p53-independent manner. ${ }^{19}$ Although ER stress could induce PUMA, it did not increase the Smad4 expression (Supplementary Figure $5 \mathrm{~h}$ ). This result suggests that there would be novel PUMA-induction mechanism, which is not related with p53 or ER stress.
Smad4 regulates PUMA through PAK1 suppression. To explore the mechanism about Smad4-induced PUMA stabilization, we performed the chemical inhibitor screening and found that AG879 could induce PUMA in serumcontaining condition (Supplementary Figure 6a). In addition, this chemical could induce Smad4 expression (Supplementary Figure 6b). As AG879 is known to be an inhibitor of PAK1, which regulates cell mobility and $G$ proteins, ${ }^{20}$ and is known to interact with Smad4, ${ }^{21}$ it seems to be a putative linker between Smad4 and PUMA. To investigate the relevance between PAK1-Smad4 or PAK1PUMA, we checked the expression of PAK1 in SF condition. In PC3 cells, SF could induce Smad4 and PUMA and suppress the PAK1 (Figure 4a). In contrast, Capan-1 did not show the reduction of PAK1 or induction of PUMA (Figure 4a). As PAK1 can be inhibited by autophosphorylation, ${ }^{4,22}$ we checked the p-PAK1 and found that SF condition could induce p-PAK1 (Figure 4b). Next, we measured the expression of PAK1 in Smad4-/- HCT116 and revealed that Smad4 could suppress PAK1 expression (Figure 4c). To reveal the effect of PAK1 on PUMA expression, 
a

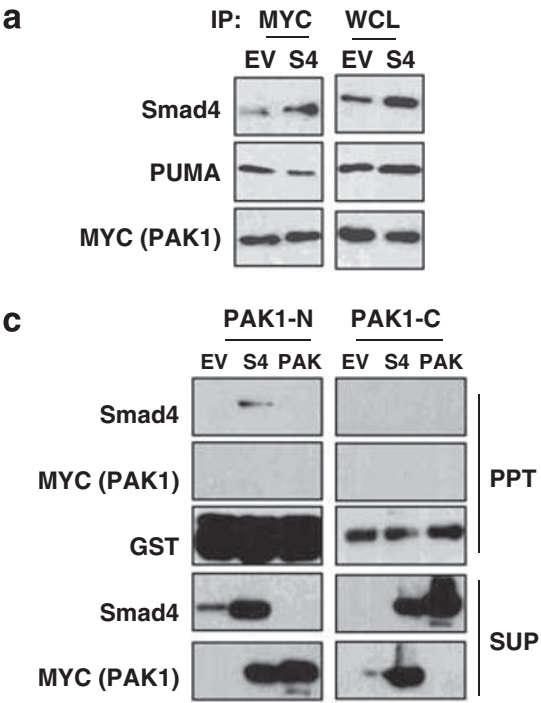

b

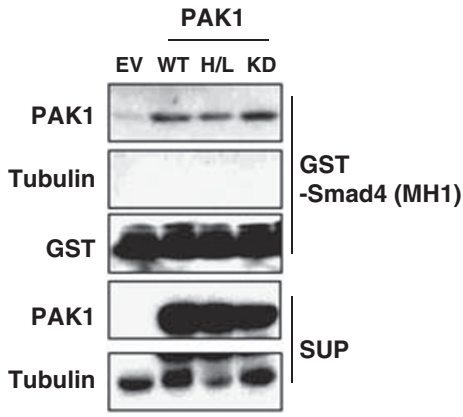

d EV WT H/L KD

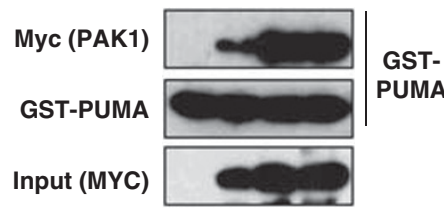

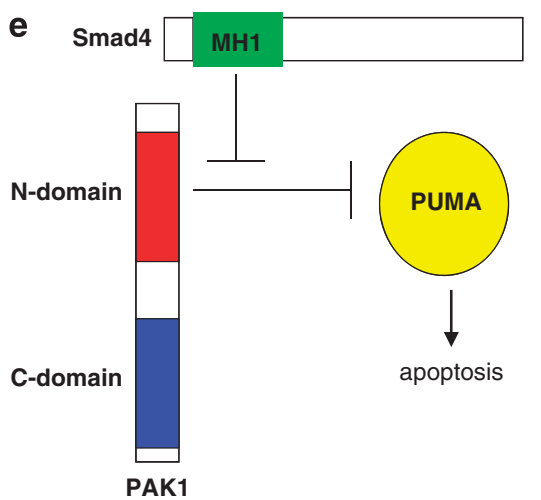

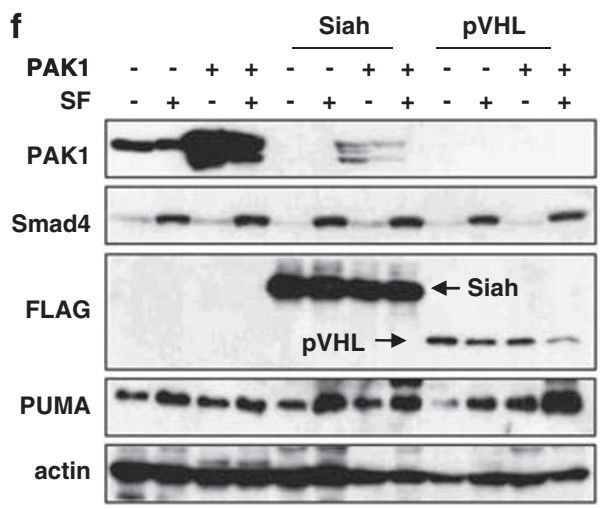

Figure 6 Direct interaction between PUMA and PAK1-Smad4. (a) PAK1 interacts with Smad4 and PUMA. HEK293 lysate overexpressing wild-type PAK1 (Myc tagged) was used for IP, which was performed with anti-Myc. PAK1 can bind to both Smad4 and PUMA, but overexpression of Smad4 attenuates PAK1 and PUMA interaction. (b) PAK1 binds to Smad4 MH1 domain. Agarose bead-conjugated GST-Smad4 (MH1) was incubated with PAK1-transfected HEK293 lysates, and Smad4-associated PAK1 was determined by WB after precipitation. Tubulin was used as a negative control. (c) N terminus of PAK1 is essential to bind to Smad4. GST pull-down was performed with PAK1 N- and C- terminal fragments. (d) Both wild and mutant PAK1 can bind to PUMA. PAK1 (Myc tagged) expressing HEK293 lysates were used for GST pull-down. (e) Interaction map between Smad4 and PAK1 and PUMA-PAK1. MH1 domain of Smad4 bound to and protected the PAK1-N-terminal domain-induced PUMA suppression. (f) Siah and pVHL reduced PAK1 but induced PUMA expression. Siah (FLAG tagged) and pVHL (FLAG tagged) were transfected to HEK293 for $24 \mathrm{~h}$ before eliminating serum from media. Expression of indicating proteins were examined by WB analysis

we transfected the Smad4 and found that Smad4 overexpression could suppress the PAK1 expression and induce PUMA, whereas PAK1 overexpression could block the PUMA induction by SF or Smad4 overexpression (Figure 4d). Instead, si-Smad4 could induce PAK1 expression (Figure 4e). We also checked the effect of mutant PAK1 on PUMA regulation. Kinase-dead PAK1 (which is point mutant at K299 residue to R) and H/L PAK1 (N-terminal G-protein binding domain mutant at $\mathrm{H} 83 \mathrm{~L}$ and $\mathrm{H} 86 \mathrm{~L}$ ) were regulated by Smad4, whereas they did not suppress the PUMA expression ${ }^{23}$ (Figure $4 \mathrm{e}$ ). In fact, PAK1$\mathrm{KD}$ and $\mathrm{H} / \mathrm{L}$ mutants did not block the SF-induced PUMA induction (Figure 4f, and Supplementary Figure 6c). Our results suggest that Smad4 induces PUMA expression through PAK1 suppression.

PAK1 regulates SF-induced cell death and PUMA expression. We next checked the effect of PAK1 on SF-induced cell death and revealed that PAK1, but not mutant
PAK1, blocked it (Figure 5a). Indeed, PAK1 could reduce the half-life of PUMA (Figure 5b). To confirm the effect of PAK1 on SF-induced cell death and PUMA induction, we measured the expression of PAK1, PUMA, and Smad4 in MIA-Paca-2, MCF-7, and MDA-MB 468 (Smad4-null cell). Interestingly, MIA-Paca-2 showed the elevated expression of PAK1 (Figure $5 c$ and Supplementary Figure $6 \mathrm{~d}$ ) and resistance to SF-induced cell death and PUMA (Figures $5 \mathrm{c}$ and $\mathrm{d}$, and Supplementary Figure 6e). Considering the result that MDAMB 468 was also resistant to SF-induced PUMA induction and cell death (Supplementary Figures $6 \mathrm{~d}$ and e), PAK1 overexpressed cells could acquire the resistance to SF-induced cell death and PUMA like Smad4-deficient condition. To confirm the role of PAK1 on PUMA regulation, we knocked down the PAK1 through siRNA and measured expression of PUMA and cell viability of MIA-Paca-2. Elimination of PAK1 could restore the PUMA induction in response to SF condition (Figure $5 \mathrm{c}$ ) and 
a

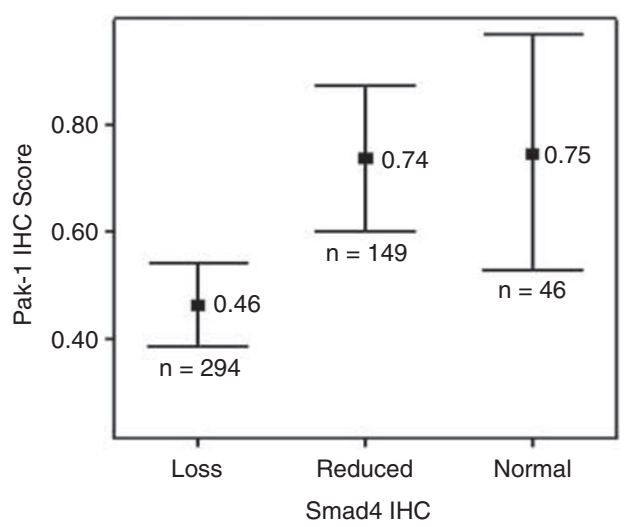

b

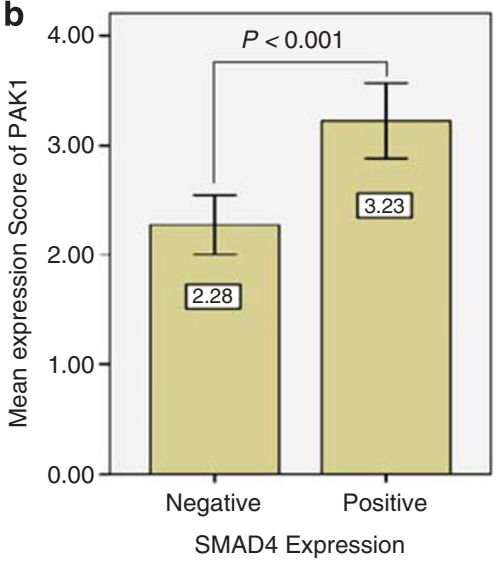

C

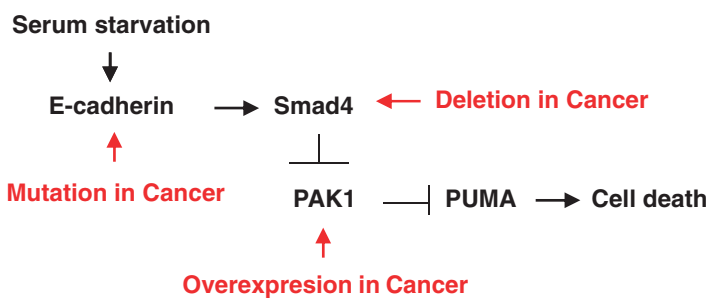

Figure 7 Elevated expression of PAK1 in Smad4-positive colon cancer tissues. (a) Analysis of PAK1 expression in 489 human colon cancer tissues using IHC analysis. In Smad4-intact tissues, PAK1 expression was elevated. (b) PAK1 expression is elevated in Smad4-negative tissues. More detail clinicopathological data were available in Supplementary Table 2. (c) Diagram for summary. In response to serum deprivation, Smad4 is induced by releasing from E-cad and suppresses PAK1-mediated PUMA reduction, which causes induction and activation of apoptosis program. Against Smad4-mediated cell death, cancer cells evoke the mutation or deletion of Smad4 itself, or mutation of E-cad, or overexpression of PAK1. Overcome mechanisms of smad4-induced cell death are depicted as red letters

promote the cell death (Figure $5 d$ ). In addition, si-PAK1 could induce PUMA expression in Smad4-deficient cell lines (Capan-1 and Panc-1; Figure 5e). These results indicate that PAK1 suppresses the PUMA expression through its enzymatic activity.

Direct interaction of Smad4-PAK1 and PAK1-PUMA. To get more detail mechanism, we performed the IP and revealed that PAK1-Smad4 pr-PAK1-PUMA could associate with each other (Figure 6a and Supplementary Figure 7a). Moreover, interaction between PAK1 and PUMA was reduced by Smad4 overexpression (Figure 6a). To confirm the direct binding between PAK1 and Smad4, we performed the GST pull-down with Smad4 $\mathrm{MH} 1$ domain $^{24}$ and found that PAK1, regardless of mutants, could associate with Smad4 (Figure 6b). We could confirm the interaction by IP analysis (Supplementary Figure 7b). Using two kinds of recombinant PAK1, PAK1-N (N-terminal G-protein binding domain; 70-149AA) and PAK1-C (kinase domain; 249-545), we could determine the PAK1-N as Smad4 binding domain (Figure $6 \mathrm{c}$ ). We could also observe the direct interaction between PAK1 and PUMA through GST pull-down and IP methods (Figure 6d). Moreover, PAK1 mutants also showed the interaction with PUMA with stronger affinity than wild-type PAK1 (Figure 6d), suggesting that enzymatic activity of PAK1 would be involved in PUMA regulation. To determine the binding domain of PAK1 with PUMA, we performed the GST pull-down again using PAK1-N and $-\mathrm{C}$ domains. Differentially from Smad4, both domains were involved in interaction with PUMA (Supplementary Figure 7c). However, when we used the endogenous PUMA, PAK1-N showed obvious interaction with PUMA (Supplementary Figure 7d), indicating that N-terminal domain of PAK1 would be more important for binding with PUMA. To confirm the binding between Smad4-PAK1-PUMA and test the binding specificity, we performed the GST pulldown again and found the specific binding of PAK1-Smad4 and PUMA-PAK1 (Supplementary Figure 7e). However, Siah-1 and pVHL did not associate with Smad4 or PUMA (Supplementary Figure 7e). In contrast, PAK1 showed the binding affinity with Siah-1 and pVHL, E3 ligase (Supplementary Figure 7f). Considering our results, Smad4 $\mathrm{MH} 1$ domain can bind to PAK1 N-terminal domain and block the PAK1-induced PUMA suppression, whereas PAK1 can suppress the PUMA expression through $\mathrm{N}$-terminal domain. Thus, Smad4, induced by SF, protected the PUMA from PAK1-induced suppression (Figure 6e).

pVHL and Siah-1 involved in Smad4-induced PAK1 suppression. Next, we checked the involvement of E3 ligases on Smad4-mediated PAK1 and PUMA regulation. Overexpression of Siah-1 could suppress the PAK1 expression, whereas si-Siah-1 could block the Smad4mediated PAK1 suppression (Supplementary Figure 8a). In addition, Siah-1 could induce PUMA expression (Supplementary Figure 8b). However, Siah-1 did not alter the SF-induced Smad4 (Supplementary Figures $8 a$ and b). Next, we checked the effect of pVHL on PUMA expression and obtained that pVHL could increase the PUMA expression (Supplementary Figure $8 \mathrm{c}$ ). To confirm this, we reperformed the similar experiment and obtained the 
same result that Siah and pVHL could suppress the PAK1 expression and enhance the PUMA response to SF condition (Figure 6f).

\begin{abstract}
Elevated expression of PAK1 in Smad4 wild-type cancers. Considering our results, functional defect of Smad4, including PAK1 overexpression, may require for survival of cancer cell in nutrient-deficient condition. To verify this, we examined the expression of Smad4 and PAK1 through IHC in 489 colon cancer tissues using tissue array (Supplementary Figures 9 and 10; clinicopathological information is available in Supplementary Table 2). In normal intestinal tissue, Smad4 was highly expressed in bottom of crypt, whereas PAK1 was highly detected in apical area of villi, reversely (Supplementary Figure 9). This result was consistent with our data that Smad4 suppressed the PAK1 expression. However, in cancer tissues, we obtained a quite different feature that PAK1 expression is increased in Smad4-positive cancers (Figures $7 \mathrm{a}$ and b). This result suggests that in human cancer PAK1 overexpression can overwhelm the Smad4-indued apoptosis, which would be a survival strategy for Smad4-positive cancer cells.
\end{abstract}

\section{Discussion}

Smad4 is a well-known TGF- $\beta$ signaling component and tumor suppressor gene. ${ }^{5,6,9}$ TGF- $\beta$ inhibits cell proliferation by regulation of $c-m y c$ and p15/INK4B, p27, and p21. Upon TGF- $\beta$, phosphorylated R-Smads (such as Smad2 and Smad3) by receptor kinase are formed the complex with Smad4 and translocated into nucleus, where Smad complex works as a transcription factor. Thus, in many kinds of human cancers genetic mutations in TGF- $\beta$ signaling components are reported. One of them is genetic mutation of Smad4, deletion or mutation of which occurs frequently in human colon and pancreatic cancers. However, TGF- $\beta$ signaling also possesses oncogenic property through promoting the EMT. ${ }^{25}$ According to recent papers, TGF- $\beta$-driven EMT is also achieved by transcriptional activity of Smads. ${ }^{26}$ Indeed, TGF- $\beta$ signaling seems to be essential for cancer metastasis, and EMT and Smad4 are core components in this signaling cascade. The question is remained how Smad4-deficient cells can enter the EMT for metastasis and Smad4 deletion or inactivation is required for TGF-b signaling inactivation. Considering that pancreatic and colon cancer shows highly aggressive phenotype and easily enter the EMT stage, there would be additional EMT-promoting mechanism. Recently, it has been suggested that Smad4 can serve as a barrier against cancer growth and metastasis in prostate cancer. ${ }^{27}$ This result is consistent with our result because nutrient or serum deprivation occurs in core region of tumor mass, ${ }^{28}$ which can be easily converted into aggressive and invasive carcinoma. Considering our result that Smad4 is significantly induced by serum starvation (Figures $1 \mathrm{a}$ and $\mathrm{b}$ ), physiological function of Smad4 would be a barrier for the too largely increasing tissue mass to be supported by the circulation system or nutrient-supplying system. However, growth of cancer cells or pre-cancerous adenoma cells is too rapid. At this point, Smad4 may suppress the cell growth by PUMAmediated apoptosis program (Figure 7c). Thus, in Smad4- intact condition, pre-cancerous cells, which are located far from blood vessel, are eliminated and cancer progression would be halted. In contrast, Smad4-deficient cells show resistance and are converted into carcinoma.

Considering the fact that defect of Smad4 occurs in $50 \%$ of pancreatic cancers, there would be additional tumorigenic pathway, which can overcome the nutrient deprivationinduced cell death, such as PAK1 overexpression (Figure 7c). In fact, elevated expression of PAK1 following cancer progression has been reported. ${ }^{29}$ We also found that PAK1 blocks the PUMA induction and SF-induced cell death (Figures 4 and 5). In addition, we could observe the induction of PAK1 in Smad4-expressed cancer tissues (Figures 7a and $b$ ). These results indicate that overexpression of PAK1 can overcome the Smad4-induced apoptosis.

In this study, we show the importance of PUMA in SFinduced cell death. Although genetic mutation of PUMA has not been reported frequently in human cancer, in a study involving an animal model, PUMA-/- mice showed sensitivity to colon cancer incidence, ${ }^{30}$ suggesting that PUMA also functions as tumor suppressor. We also showed the involvement of Siah-1 and PVHL in PAK1-PUMA regulation (Figure 6f). Considering the previous reports that $\mathrm{pVHL}$ is frequently deleted in human renal cell carcinoma ${ }^{31}$ and Siah-1 possesses tumor suppressor potentials, ${ }^{32}$ genetic or epigenetic defects in pVHL or Siah-1 may also enhance the PAK1 expression and weaken the Smad4-PUMA axis. Although more accumulated evidences are required, reduction of $\mathrm{pVHL}$ or Siah-1 would be one of the reasons for elevated expression of PAK1 in human cancer, which may lead to disruption of Smad4-induced cell death.

Collectively, human epithelial cells, located far from blood vessel or nutrients, are eliminated by Smad4-PUMA system, which can be overcome in cancer by Smad4 deletion or PAK1 overexpression. These results will provide the basis for development of smart anti-cancer drug such as specific inhibitor of PAK1 and PUMA.

\section{Materials and Methods}

Cell lines and reagents. Cell lines used in this research were obtained from the American Type Culture Collection (Manassas, VA, USA) or Coriell Cell Repositories (normal fibroblast; GM00038, Coriell Cell, Camden, NJ, USA) and maintained in RPMI-1640, DMEM, or MEM containing $10 \%$ FBS and antibiotics. For serum starvation, cell lines were incubated with SF media for indicating times. General reagents were provided by Sigma (St. Louis, MO, USA). Chemical inhibitors were purchased from Calbiochem (St. Louis, MO, USA). IGF-1 and TGF$\beta$-neutralizing antibody were obtained from R\&D Systems (Minneapolis, MN, USA).

Western blot analysis, IP, and GST pull-down assays. Proteins are extracted from cells by radioimmunoprecipitation assay (RIPA) buffer. Samples were applied to SDS-PAGE, and western blotting (WB) analysis was performed by means of general protocol. Blotted membranes were incubated with primary antibodies detecting Smad4 (B-8; 1/1000; Santa Cruz Biotechnology, Santa Cruz, CA, USA), PUMA (PC686; 1/5,00; Calbiochem), PAK1 (2602; 1/1000; Cell Signaling Technology, Danvers, MA, USA), p-PAK1 (2605; 1/1000; Cell Signaling Technology), actin (I-19; 1/1000; Santa Cruz Biotechnology), E-cadherin (G-10; 1/500; Santa Cruz Biotechnology), $\alpha$-tubulin (TU-02; 1/1000; Santa Cruz Biotechnology), FLAG (F3165; 1/1000; Sigma), Myc (clone 9E10; 1/1000; Sigma), p53 (DO-1; 1/5000; Santa Cruz Biotechnology), p21 (2946; 1/1000; Cell Signaling Technology), GST (B-14; 1/1000; Santa Cruz Biotechnology), and Cox-2 (C-20; 1/1000; Santa Cruz Biotechnology). Secondary antibodies were HRP-linked goat anti-mouse, goat anti-rabbit, and mouse anti-goat antibodies (all 1/10000; Pierce, Thermo Fisher Scientific Inc., Rockford, IL, USA). The whole-cell lysates 
were incubated first with proper antibodies for $4 \mathrm{~h}$ at $4^{\circ} \mathrm{C}$ and then with protein $\mathrm{A} / \mathrm{G}$ agarose beads (Invitrogen, Carlsbad, CA, USA) for $2 \mathrm{~h}$. After centrifugation and washing with phosphate-buffered saline (PBS) and RIPA, the immunocomplexes were applied to SDS-PAGE and WB analysis. To determine the direct interaction between proteins, agarose bead-conjugated GST-Smad4 (MH1), PUMA, PAK1-N/ $\mathrm{C}$ fragment, and E-cad were incubated with cell lysates for $2 \mathrm{~h}$ at $4^{\circ} \mathrm{C}$. Through the same procedure with IP, precipitated proteins were determined by WB analysis. More detail procedure for IP, WB, and GST pull-down are available in our previous literatures. ${ }^{7,8}$

Recombinant proteins. To produce the recombinant proteins, DNA fragments encoding human PAK1 N-terminal domain (residues 70-149) and Cterminal domain (residues 249-545) were ligated into the EcoRI and Hindll sites of the PGEX-TEV vector, which is a modified vector made by adding a TEV protease cleavage site to pGEX-4T1 (Invitrogen). Domains were determined according to previous literature. ${ }^{4}$ The full-length human PUMA (residues 1-193) also comprised the same enzyme sites as the PGEX-TEV vector. The recombinant proteins were expressed in the $E$. coli strain BL21 (DE3) as GST-fusion proteins. The proteins were purified by glutathione-affinity chromatography.

Transfection of mammalian expression plasmids and siRNA. The mammalian expression plasmid encoding MH1-Smad4, ${ }^{24}$ PUMA, PUMA-Luc, ${ }^{14}$ wild and mutant type of PAK $1,{ }^{23}$ E-cad, and DN-E-cad ${ }^{33}$ were obtained from Addgene (Cambridge, MA, USA). For in vitro gene knockout, siRNAs against target proteins were generated following previous literatures. Target sequence of siRNA for each gene is described below: Smad4: $5^{\prime}-\mathrm{CA}$ TTGGATGGGAGGC-3'; PUMA: 5'-TCTCATCATGGGACTCCTG-3'; PAK1 no. 1: 5'-TCTGTATACACACGGTCTG-3'; PAK1 no. 2: 5'-GGAGAAATTACGAAGCATA-3'; Smad2: 5'-AATGGAGTGAGTATAGTCATCCAGA-3'; Smad3: 5'-AGATCTTCA GGTTGCATCCTGGTGG-3'; Smurf: 5'-GATTCGAACCTTGCAAAGAAAGAC-3'; FAK1: 5'-GCATGTGGCCTGCTATGGA-3'; Smad7: 5'-AAGCTCAATTCGGACAA CAAG-3; Siah-1: 5'-GATAGGAACACGCAAGCAA-3'; and VHL: 5'-AACTGAATT ATTTGTGCCATC-3'. Transfection was performed for $24 \mathrm{~h}$ using Jei-pei reagent (JetPEl; Polyplus Transfection, New York, NY, USA) according to the manufacturer's protocol.

Luciferase assay. To address the PUMA activity, PUMA-Luc vector was transfected to cells for $24 \mathrm{~h}$, and cells were incubated in SF condition for $6 \mathrm{~h}$. After washing with wash buffer (Promega, Madison, WI, USA), cells were lysed by lysis buffer. Luciferase activity was determined by luminometer.

Measurement of cell viability and proliferation. MTT assay was performed to examine cell viability. Cells were incubated in $0.5 \mathrm{mg} / \mathrm{ml}$ of MTT solution for $4 \mathrm{~h}$ at $37^{\circ} \mathrm{C}$. After removing excess solution, precipitated materials were dissolved in $200 \mu \mathrm{l}$ dimethyl sulfoxide and quantified by measuring absorbance at $540 \mathrm{~nm}$.

Immunofluorescence staining. Cells grown on coverslips were washed and fixed with $100 \% \mathrm{Me}-\mathrm{OH}$ for $20 \mathrm{~min}$. After blocking with buffer (PBS/ anti-human $A b(1: 500))$ to eliminate nonspecific binding, cells were incubated with primary antibodies $\left(1: 200 ;\right.$ OVN at $\left.4{ }^{\circ} \mathrm{C}\right)$ and FITC-conjugated secondary antibodies at $37^{\circ} \mathrm{C}$ for $1 \mathrm{~h}$. Nuclei were stained with $4^{\prime} 6$-diamidino-2-phenylindole. After a final wash with PBS three times, coverslips were mounted with mounting solution (H-5501; Vector Laboratories, Burlingame, CA, USA).

Patients and tissue samples. Our study enrolled a consecutive series of 489 patients with colorectal adenocarcinoma. All patients were diagnosed and treated at the Hanyang University Hospital (Seoul, Korea) between January 1991 and August 2001. There were 272 male and 217 female patients. The mean age of patients was 58 years. The tumors consisted of 463 non-mucinous adenocarcinomas and 26 mucinous adenocarcinomas.

Tissue microarray construction. Tissue microarrays (TMAs) were constructed from archival formalin-fixed, paraffin-embedded tissue blocks using a manual tissue arrayer (Quick-Ray Manual Tissue Microarrayer, Unitma Co. Ltd, Seoul, Korea). As described previously, ${ }^{29,34}$ for each sample, areas rich in tumor cells were identified by light microscopic examination of hematoxylin-eosin-stained sections and then selected for use in TMAs. Tissue cylinders with a diameter of $2 \mathrm{~mm}$ were punched from the previously marked tumor area of each block (donor block) and then transferred to a recipient paraffin block. This resulted in a $6 \times 10$ array for 60 cases.

Immunohistochemical staining. For immunohistochemical staining, multiple $4 \mu \mathrm{m}$ sections were cut with a Leica microtome (Wetzlar, Germany). Sections were transferred to adhesive-coated slides. TMA slides were dewaxed by heating at $55^{\circ} \mathrm{C}$ for $30 \mathrm{~min}$ and by three washes, $5 \mathrm{~min}$ each, with xylene. Tissues were rehydrated by a series of 5 min washes in $100,90,70 \%$ ethanol, and PBS. Antigen retrieval was performed by microwaving the samples for $4 \mathrm{~min} 20 \mathrm{~s}$ at full power in $250 \mathrm{ml}$ of $10 \mathrm{mM}$ sodium citrate ( $\mathrm{pH} \mathrm{6.0)}$. Endogenous peroxidase activity was blocked with $0.3 \%$ hydrogen peroxidase for $20 \mathrm{~min}$. The primary mouse monoclonal Smad4 antibody (B-8; Santa Cruz Biotechnology) was diluted 1:250. The primary rabbit polyclonal PAK1 antibody (2602; Cell Signaling Technology) was diluted 1:50. Then, they were incubated at room temperature for $1 \mathrm{~h}$. After three washes, 2 min each with PBS, the sections were incubated with a biotinylated goat anti-mouse secondary antibody for $30 \mathrm{~min}$ (DAKO, Glostrup, Denmark). After three washes, 2 min each with PBS, horseradish peroxidase-streptavidin (DAKO) was added to the section for $30 \mathrm{~min}$, followed by another three washes, 2 min each with PBS. The samples were developed with 3,3'-diaminobenzidine substrate (Vector Laboratories) for $1 \mathrm{~min}$ and counterstained with Mayer's hematoxylin. Then, the slides were dehydrated following a standard procedure and sealed with coverslips. For negative control, Smad4 and PAK1 antibodies omitted during the primary antibody incubation.

Interpretation of Smad4 and PAK1 immunostainings. Smad4 and PAK1 expression was evaluated semiquantitatively by two independent pathologists (Jang SM and Paik SS) without prior knowledge of the clinical follow-up data for each case. On examination, each case was scored as having normal, reduced, or loss of SMAD4 expression. Normal nuclear staining in the epithelial cells lining normal crypts or inflammatory cells in the mesenchymal stroma on the same section served as an internal control (i.e., normal expression refers to the same expression as seen in these control cells). Loss of expression was defined as absence of nuclear staining. Reduced expression was graded when a weaker expression, but not a complete absence of nuclear staining, was noted compared with the control cells. The interpretation of cytoplasmic PAK1 expression was based on staining intensity and staining extent. Staining intensity for PAK1 was scored as 0 (negative), 1 (weak), 2 (moderate), and 3 (strong). Staining extent was scored as $0(0 \%), 1(1-25 \%), 2(26-50 \%), 3(51-75 \%)$, and $4(76-100 \%)$ according to the percentage of positively stained cells.

Statistical analysis. Statistical analysis was performed using SPSS software (version 15.0, SPSS, Chicago, IL, USA). The $\chi^{2}$-test for linear trend and one-way ANOVA test were used to examine the association between Smad4 and PAK1 and clinicopathological parameters, including age, gender, tumor location, tumor size, tumor type, T category, N category, AJCC stage, Dukes stage, and differentiation. The Kaplan-Meier method was used to calculate overall survival and disease-free survival curves. Univariate survival analysis with the log-rank test was used to compare the difference between the survival rates of the patient subgroups. Multivariate survival analysis with the Cox proportional hazards regression model was used to evaluate the independent prognostic factors. A difference of $P<0.05$ between groups was considered significant.

\section{Conflict of Interest}

The authors declare no conflict of interest.

Acknowledgements. This research was supported by Basic Science Research Program through the National Research Foundation of Korea funded by the Ministry of Education, Science and Technology (2010-0022413).

1. Weinberg RA. How cancer arises. Sci Am 1996; 275: 62-70.

2. Vogelstein B, Kinzler KW. Cancer genes and the pathways they control. Nat Med 2004; 10: 789-799.

3. Bardeesy N, DePinho RA. Pancreatic cancer biology and genetics. Nature Rev Cancer 2002; 2: 897-909.

4. Lei M, Lu W, Meng W, Parrini MC, Eck MJ, Mayer BJ et al. Structure of PAK1 in an autoinhibited conformation reveals a multistage activation switch. Cell 2000; 102: 387-397. 
5. Hahn SA, Schutte M, Hoque AT, Moskaluk CA, da Costa LT, Rozenblum E et al. DPC4, a candidate tumor suppressor gene at human chromosome 18q21.1. Science 1996; 271: 350-353.

6. Gallione CJ, Repetto GM, Legius E, Rustgi AK, Schelley SL, Tejpar S et al. A combined syndrome of juvenile polyposis and hereditary haemorrhagic telangiectasia associated with mutations in MADH4 (SMAD4). Lancet 2004; 363: 852-859.

7. Lee SH, Lee SJ, Jung YS, Xu Y, Kang HS, Ha NC et al. Blocking of p53-Snail binding, promoted by oncogenic K-Ras, recovers p53 expression and function. Neoplasia 2009; 11 22-31.

8. Lee SH, Shen GN, Jung YS, Lee SJ, Chung JY, Kim HS et al. Antitumor effect of novel small chemical inhibitors of Snail-p53 binding in K-Ras-mutated cancer cells. Oncogene 2010; 29: 4576-4587.

9. Massague J, Blain SW, Lo RS. TGF- $\beta$ signaling in growth control, cancer, and heritable disorders. Cell 2000; 103: 295-309.

10. Chen ZY. Cell cycle, differentiation and regeneration: where to begin? Cell Cycle 2006; 5 : 2609-2612.

11. Zavadil J, Böttinger EP. TGF-beta and epithelial-to-mesenchymal transitions. Oncogene 2005; 24: 5764-5774.

12. Wu WS. The signaling mechanism of ROS in tumor progression. Cancer Metastasis Rev 2006; 25: 695-705.

13. Nakano K, Vousden KH. PUMA, a novel proapoptotic gene, is induced by p53. Mol Cell 2001; 7: 683-694.

14. Yu J, Zhang L, Hwang PM, Kinzler KW, Vogelstein B. PUMA induces the rapid apoptosis of colorectal cancer cells. Mol Cell 2001; 7: 673-682.

15. Zhou S, Buckhaults P, Zawel L, Bunz F, Riggins G, Dai JL et al. Targeted deletion of Smad4 shows it is required for transforming growth factor $b$ and activin signaling in colorectal cancer cells. Proc Natl Acad Sci USA 1998; 95: 2412-2416.

16. Oda T, Kanai Y, Oyama T, Yoshiura K, Shimoyama Y, Birchmeier W et al. E-cadherin gene mutations in human gastric carcinoma cell lines. Proc Natl Acad Sci USA 1994; 91: 18581862.

17. Melki JR, Vincent PC, Brown RD, Clark SJ. Hypermethylation of E-cadherin in leukemia Blood 2000; 95: 3208-3213.

18. Yu J, Wang Z, Kinzler KW, Vogelstein B, Zhang L. PUMA mediates the apoptotic response to p53 in colorectal cancer cells. Proc Natl Acad Sci USA 2003; 100: 1931-1936.

19. Galehdar Z, Swan P, Fuerth B, Callaghan SM, Park DS, Cregan SP. Neuronal apoptosis induced by endoplasmic reticulum stress is regulated by ATF4-CHOP-mediated induction of the Bcl-2 homology 3-only member PUMA. J Neurosci 2010; 30: 16938-16948.

20. Dummler B, Ohshiro K, Kumar R, Field J. Pak protein kinases and their role in cancer. Cancer Metastasis Rev 2009; 28: 51-63.

21. Barrios-Rodiles M, Brown KR, Ozdamar B, Bose R, Liu Z, Donovan RS et al. Highthroughput mapping of a dynamic signaling network in mammalian cells. Science 2005 ; 307: $1621-1625$.
22. Weisz Hubsman M, Volinsky N, Manser E, Yablonski D, Aronheim A. Autophosphorylationdependent degradation of Pak1, triggered by the Rho-family GTPase, Chp. Biochem J 2007; 404: 487-497.

23. Sells MA, Knaus UG, Bagrodia S, Ambrose DM, Bokoch GM, Chernoff J. Human p21activated kinase (Pak1) regulates actin organization in mammalian cells. Curr Biol 1997; 7 : 202-210.

24. Zawel L, Dai JL, Buckhaults $P$, Zhou S, Kinzler KW, Vogelstein B et al. Human Smad3 and Smad4 are sequence-specific transcription activators. Mol Cell 1998; 1: 611-617.

25. Ozdamar B, Bose R, Barrios-Rodiles M, Wang HR, Zhang Y, Wrana JL. Regulation of the polarity protein Par6 by TGFbeta receptors controls epithelial cell plasticity. Science 2005; 307: 1603-1609.

26. Xu J, Lamouille S, Derynck R. TGF- $\beta$-induced epithelial to mesenchymal transition. Cell Res 2009; 19: 156-172.

27. Ding Z, Wu CJ, Chu GC, Xiao Y, Ho D, Zhang J et al. SMAD4-dependent barrier constrains prostate cancer growth and metastatic progression. Nature 2011; 470: 269-273.

28. Bergers G, Benjamin LE. Tumorigenesis and the angiogenic switch. Nat Rev Cancer 2003; 3: $401-410$

29. Carter JH, Douglass LE, Deddens JA, Colligan BM, Bhatt TR, Pemberton JO et al. Pak-1 expression increases with progression of colorectal carcinomas to metastasis. Clin Cancer Res 2004; 10: 3448-3456.

30. Qiu W, Carson-Walter EB, Kuan SF, Zhang L, Yu J. PUMA suppresses intestinal tumorigenesis in mice. Cancer Res 2009; 69: 4999-5006.

31. Ma X, Yang K, Lindblad P, Egevad L, Hemminki K. VHL gene alterations in renal cell carcinoma patients: novel hotspot or founder mutations and linkage disequilibrium. Oncogene 2001; 20: 5393-5400.

32. Roperch JP, Lethrone F, Prieur S, Piouffre L, Israeli D, Tuynder M et al. SIAH-1 promotes apoptosis and tumor suppression through a network involving the regulation of protein folding, unfolding, and trafficking: identification of common effectors with p53 and p21(Waf1). Proc Natl Acad Sci USA 1999; 96: 8070-8073.

33. Onder TT, Gupta PB, Mani SA, Yang J, Lander ES, Weinberg RA. Loss of E-cadherin promotes metastasis via multiple downstream transcriptional pathways. Cancer Res 2008; 68: $3645-3654$.

34. Langeveld D, van Hattem WA, de Leng WW, Morsink FH, Ten Kate FJ, Giardiello FM et al. SMAD4 immunohistochemistry reflects genetic status in juvenile polyposis syndrome. Clin Cancer Res 2010; 16: 4126-4134.

Cell Death and Disease is an open-access journal published by Nature Publishing Group. This work is licensed under the Creative Commons Attribution-Noncommercial-No Derivative Works 3.0 Unported License. To view a copy of this license, visit http://creativecommons.org/licenses/by-nc-nd/3.0/

\section{Supplementary Information accompanies the paper on Cell Death and Disease website (http://www.nature.com/cddis)}

